\title{
A Study on the Prevalence and Microbiological Profile of Nosocomial Infections in the ICU of a Tertiary Care Hospital in Eastern India
}

\author{
Pampita Chakraborty $^{1 *}$ and Sukumar Mukherjee ${ }^{2}$ \\ ${ }^{1}$ Institute of Post Graduate Medical Education \& Research, Kolkata, India \\ ${ }^{2}$ MD Medicine, FRCP, FRCP, GD Hospital \& Diabetes Institute, Kolkata, India \\ *Corresponding author
}

\section{Keywords}

Nosocomial

Infections,

Drug Sensitivity,

Antibiotic

Resistance, Intensive Care

Unit.

\begin{tabular}{l}
\hline Article Info \\
\hline Accepted: \\
22 April 2016 \\
Available Online: \\
10 May 2016
\end{tabular}

\section{A B S T R A C T}

This study was done to determine the prevalence of Nosocomial infections in the ICU and to identify the common microorganisms causing these infections and their antimicrobial sensitivity pattern. Nosocomial infection or hospital-acquired infection is a localized or a systemic condition resulting from an adverse reaction to the presence of infectious agents. Nosocomial infections are not present or incubating when the patient is admitted to hospital or other health care facility. They are caused by pathogens that easily spread through the body. Many hospitalized patients have compromised immune systems, so they are less able to fight off infections. These infections occur worldwide, both in the developed and developing countries. They are a significant burden to patients and public health. They are a major cause of death and increased morbidity in hospitalized patients, which is a matter of serious concern today. This study was done during the period of one year (2013-2014) in the ICU of the tertiary care hospital in eastern India. Prevalence of nosocomial infection was determined; site of infection and the pattern of microorganisms were identified along with the assessment of antibiotic susceptibility profile. Patients who developed an infection after 48 hours of admission to the ICU were included in the study. A total of 324 ICU patients were analyzed, of these 79 patients were found to have developed a nosocomial infection (24.3\% prevalence). Urinary tract infection was found to be more predominant followed by respiratory tract infection and soft tissue infection. The most frequently isolated microorganism was Escherichia coli, Pseudomonas aeruginosa, Acinetobacter, Klebsiella pneumoniae followed by other organisms respectively. Antibiotic susceptibility test of these isolates was done against commonly used antibiotics. Patients admitted to the ICU are especially susceptible to nosocomial infections. Despite adequate antimicrobial treatment, nosocomial ICU infections can significantly affect ICU stay and can cause an increase in patient's morbidity and mortality. Adherence to infection protocol, proper monitoring and the judicious use of antibiotics are important in preventing such infections on a regular basis. 


\section{Introduction}

Nosocomial infection, also called healthcare acquired infection, is defined by the CDC as a localized or systemic condition resulting from an adverse reaction to the presence of an infectious agent(s) or toxin(s), without any evidence that the infection was present or incubating at the time of admission to the acute care setting. (Garnes et al., 1988) Patients admitted to the ICU are mostly susceptible to nosocomial infections in view of significant risk factors such as central venous catheterization, urinary catheterization, mechanical ventilation, stress ulcer prophylaxis and increasing length of ICU stay. (Vincent et al., (1995), Ak et al., (2011), Esen et al., (2004), Legras et al., (1998).

Throughout the world multi-drug resistant nosocomial infections are one of the leading causes of death and morbidity amongst hospitalized patients, accounting a major burden on the patients and public health system of any country. (Shehabi et al., (1996), Ducel et al.,(2002), Krishna et al.,) Critically ill Intensive care unit (ICU) patients are most vulnerable for developing these infections (Barai et al., 2010). Compared with an average patient, an ICU patient has five to seven folds higher risk of nosocomial infection and ICU infections contributes to $20 \%$ to $25 \%$ of all nosocomial infections in a hospital (Günserena et al., 1999). Factors like increasing use of invasive devices, immunosuppressive drugs and status as well as irrational use of antibiotic therapy in ICUs are all contributing for the same. (Zhang et al.,). The patterns of microorganisms causing various infections and their antibiotic resistance pattern vary widely from one country to another; as well as from one hospital to other and even among ICUs within one hospital (Barai et al., 2010).

During the last few years the increase in antibiotic resistance has compromised the selection of empirical complicated treatment. Better management of patients cannot be ensured and the antibiotic policy cannot be designed if the profile of prevalent organisms along with their antimicrobial resistance pattern is known. Therefore, the present study was planned with an objective to evaluate the microbiological profile and their antimicrobial resistance pattern rate among different infections in the ICU patients in tertiary care hospital in Kolkata, India.

\section{Material and Methods}

The study was conducted in the ICU of GD Hospital \& Diabetes Institute, a tertiary care hospital in Kolkata. Sample of the patient admitted in the ICU during the period of September 2013 to May 2014 were collected.

The CDC for disease control and prevention define ICU associated infection as those that occur after 48 hours of ICU admission or within 48 hour after transfer from an ICU. In the present study patients who had developed infection after 48 hour of admission to the ICU or clinically suspected of having acquired any infection were included in the study. Patient showing clinical signs of infection on or prior to admission or transfer to ICU were not included.

Few clinical signs and symptoms suggestive of infections are as follows: fever $>38^{\circ} \mathrm{C}$, New infiltrates on chest X-ray, Persistent Tracheal aspirates/secretions, Leukocytosis $>10000 / \mathrm{mm} 3$, Turbid urine, Suprapubic tenderness, Thrombophlebitis, Cloudy effluent containing more than 100 Polymorphonuclear cells/mm3, Abdominal pain or tenderness, Dysuria, Burning micturition.

Various sources of clinical specimens included blood, urine, pus, cerebrospinal fluid (CSF), catheter tips, endotracheal tips, bronchial aspirates, central venous catheters (CVC) etc. 
All the organisms were identified morphologically and biochemically by standard laboratory procedure. Specimens received were plated on the blood agar and Mac and incubated aerobically overnight at 37 degree centigrade. Single and mixed growth (two or more than two isolates) per specimens isolated from all the sample were identified by observing the colony characteristics on blood agar, Mac-Conkey agar plates and biochemical reactions using standard microbiological methods.

Only bacterial nosocomial infections were studied in detail in present study. Candida sp. was also identified. Samples were subjected to the testing and antibiotic sensitivity.

The following antibiotics (Hi-Media disc in $\mathrm{mcg}$ ) were tested for sensitivity: Amikacin, Cefoperazone+Sulbactum, Ampicillin, Ampicillin+Sulbactum, Piperacillin +Tazobactum, Gatifloxacin, Cefazolin, Imipenam, Cefuroxime, Gentamycin, Cefotaxime.

Other information regarding the patient including age, gender, date of admission, was also collected from the case records of the patients.

The collected data was compiled and analysis was done using Statistical Package for Social Sciences (SPSS), version 20 (IBM, Chicago, USA). The results were expressed using appropriate statistical methods.

\section{Results and Discussion}

During the period of the study, a total of 324 medical ICU patients records were reviewed, of these 79 patients were found to develop nosocomial infection (24.3\%). Out of 79 cases that developed Nosocomial Infection, urinary tract infection was the commonest $42(53.43 \%)$, followed by respiratory infection $24(30.38 \%)$ and soft tissue infection including surgical site 13(15.19\%).

(Table 1). Gram-negative organisms were more common $(86.67 \%)$ than the grampositive organism (13.33\%).

Urinary tract infection were frequently caused by E.coli (38.40\%) followed by Pseudomonas aeruginosa (23.10\%), Acinetobacter (14\%), Enterococcus (13\%) \& Klebsiella (11.50\%). E.coli isolates were sensitive to Gentamycin \& Nitrofuratoin. Pseudomonas isolates were sensitive to Imipenem, Piperacillin, Gentamycin \& Amikacin.

Respiratory Tract Infection was most frequently caused by Acinetibacter (40\%) followed by Pseudomonas (29.80\%), Klebsiella (21.70\%) and E.coli (8.50\%). (Table 2)

$24 \%$ of Acinetobacter isolates were MDRO; the other were sensitive to Imipenem, Piperacillin, Polymixin, Cefoperazone, Colistin, Teteracyclin \& Doxycyclin. E.coli isolates were sensitive to Gentamycin, Amikacin \& Imipenem.

The average ICU stay of patients with and without nosocomial infection was 15.7days (4-40days) and 5.2 days (3-21days) (Table 3)

Our study showed the prevalence of Nosocomial infection, its type, antibiotic susceptibility pattern of bacterial organism isolated from different samples from critically ill patient's after 48hours of admission to detect the Hospital acquired infection.

In this study the prevalence of Nosocomial Infection was $24.3 \%$. The most predominant organism in our study was E.coli $(34.6 \%)$ followed by Pseudomonas (32.6\%), Acinetobacter (13.9\%), Klebsiella (12.1\%), \& Enterococcus (5\%). (Table 4) 
Table.1 Prevalence of Nosocomial Infections

\begin{tabular}{|c|c|c|}
\hline $\begin{array}{c}\text { Nosocomial Infection } \\
\text { (site) }\end{array}$ & Number & Percentage \\
\hline Urinary Infections & 42 & $53.43 \%$ \\
\hline Respiratory Infections & 24 & $30.38 \%$ \\
\hline $\begin{array}{c}\text { Soft tissue/surgical site } \\
\text { infections }\end{array}$ & 13 & $15.19 \%$ \\
\hline Total & 79 & \\
\hline
\end{tabular}

Table.2 Patterns of Microorganisms

\begin{tabular}{|l|l|l|l|l|l|}
\hline \multicolumn{2}{|c|}{ UTI } & \multicolumn{2}{c|}{ RTI } & \multicolumn{2}{c|}{$\begin{array}{l}\text { Soft Tissue/surgical } \\
\text { site }\end{array}$} \\
\hline Organism & $\%$ & Organism & $\%$ & Organism & $\%$ \\
\hline E. coli & 38.4 & Acinetobacter & 40 & Proteus & 25.9 \\
\hline Pseudomonas & 23.1 & Pseudomonas & 29.8 & E. coli & 24.7 \\
\hline Acinetobacter & 14 & Klebsiella & 21.7 & S.aureus & 21 \\
\hline Enterococcus & 13 & E. coli & 8.5 & Pseudomonas & 8.6 \\
\hline Klebsiella & 11.5 & & & Klebsiella & 7.4 \\
\hline
\end{tabular}

Table.3 Average Duration of ICU stay

\begin{tabular}{|l|l|}
\hline Patient Group & No. of days \\
\hline $\begin{array}{l}\text { Study Group (Patients with Nosocomial } \\
\text { Infections) }\end{array}$ & 15.7 days \\
\hline Matched Control Group & 5.2 days \\
\hline (Patients without Nosocomial Infections) & \\
\hline
\end{tabular}

Table.4 Causative Organisms for Nosocomial Infections

\begin{tabular}{|l|c|}
\hline \multicolumn{1}{|c|}{ Organism } & Percentage \\
\hline E. coli & $34.60 \%$ \\
\hline Pseudomonas & $32.60 \%$ \\
\hline Acinetobacter & $13.90 \%$ \\
\hline Klebsiella & $12.1 \%$ \\
\hline Enterococcus & $5 \%$ \\
\hline Candida & $1.70 \%$ \\
\hline
\end{tabular}


Table.5 Antibiotic Sensitivity and Resistance Pattern of Different Microorganisms

\begin{tabular}{|l|c|c|c|c|}
\hline \multicolumn{1}{|c|}{ Antibiotics } & \multicolumn{4}{c|}{ Percentage (\%) } \\
\hline & $\boldsymbol{E}$ coli & Pseudomonas & Acinetobacter & Klebsiella \\
\hline Imipenem & 100 & 100 & 88 & \\
\hline Meropenem & 100 & 62 & 88 & \\
\hline Polymixin B & 100 & & 100 & \\
\hline Amikacin & 78 & 23 & 40 & \\
\hline Gentamicin & 78 & 23 & 40 & \\
\hline Cepefime & 40 & 20 & 20 & \\
\hline Levofloxacin & 38 & 42 & 60 & \\
\hline Amp Sulbactum & 18 & & 22 & \\
\hline Linezolid & & & & 100 \\
\hline Vancomycin & & & & 96 \\
\hline Teicoplanin & & & & 44 \\
\hline Co-Trimoxazole & & & & 38 \\
\hline Clindamycin & & & & 25 \\
\hline Moxifloxacin & & & & \\
\hline Piperacillin $+\mathrm{Tz}$ & 40 & & & \\
\hline
\end{tabular}

A predominance of gram-positive organism was observed in our study. It was also observed that $24 \%$ Acinetobacter species were MDRO and ESBL production was noted in $42 \%$ isolates. In this study high level of resistance was observed in Ciprofloxacin (82.67\%), Gentamycin (60.20\%) and Piperacillin $(52.33 \%)$ against the common isolates E.coli and Pseudomonas aeruginosa. High resistance to Cephalexin, Cephalosporin \& Quinolones amongst E.coli and P.aeruginosa was observed, which was in concordance to other studies from the northern part of India. This shows that the isolates were resistant to the commonly used antibiotics used in the hospital \& these findings may be attributed to extensive usage of Cephalosporin \& Quinolones in the hospitals.

It has been observed that Amikacin, Meropenem showed good sensitivity against all bacterial isolate from the ICU. Gram positive isolates were $100 \%$ sensitive to Linezolid and Vancomycin. (Table 5)

In conclusion, a sustained coordination between the intensivist and the clinical microbiologist is essential not only for improving clinical outcome but also for optimizing resource utilization. Appropriate antibiotic utilization in ICU is crucial not only to ensure an optimal outcome, but also to prevent the emergence of multi drug resistance. Antibiotic policies, effective surveillance, and scrutiny of epidemiological trends of these infections are need of the hour for better management of ICU infections with resistant organisms. There is an evident necessity to study not only the trends in epidemiology of nosocomial infections but also the local situations for which multicenter studies need to be carried out in our country to coordinate and arrive at protocols based on certain patterns of antibiotic resistance. Alteration and rotation in antibiotic 
prescribing patterns would decline the antibiotic resistance.

\section{Acknowledgement}

We are thankful to the laboratory staff and paramedical personnel of GD Hospital and Diabetes Institute Kolkata for their cooperation and sincere thanks to all those who willingly participated in the present study.

\section{References}

Garnes, J.S., Jarvis, W.R., Emori, T.G., Horan, T.C., Hughes, J.M., C.D.C. 1988. Definitions for nosocomial infections. Am. J. Infect. Control, 16: 128-140.

Vincent, J.L., Bihari, D.J., Suter, P.M., Bruining, H.A., White, J., Nicolas-Chanoin, M.H., Wolff, M., Spencer, R.C., Hemmer, M. 1995. The prevalence of nosocomial infection in intensive care units in Europe. Results of theEuropean Prevalence of infection in Intensive Care (EPIC) Study. EPIC International Advisory Committee. JAMA, 274: 639-644.

Ak, O., Batirel, A., Ozer, S., Colakoglu, S. 2011. Nosocomial infections and risk factors in the intensive care unit of a teaching and research hospital: A prospective cohort study. Med. Sci. Monit., 17: PH29-34.

Esen, S., Leblebicioglu, H. 2004. Prevalence of nosocomial infections at Intensive care units in Turkey: A multicentre 1-day point prevalence study. Scan. J. Infect. Dis., 36: 144-8.

Legras, A., Malvy, D., Quinioux, A.I., Villers, D., Bouachour, G., Robert, R., Thomas, R.
1998. Nosocomial infections: prospective survey of incidence in five French intensive care units. Intensive Care Med., 24: 1040-6.

Shehabi, A.A., Baadran, I. 1996. Microbial infection and antibiotic resistance patterns among Jordanian intensive care patients. Eastern Mediterranean Health J., 2(3): 515520.

Ducel, G., Fabry, J., Nicolle, L. 2002. editors. Prevention of hospitalacquired infections: A practicle guide.2nd ed. Geneva, World Health Organization.

Krishna, Prakash, S. Nosocomial Infections: An overview. New Delhi: Maulana Azad Medical College. 13p.

Barai, L., Fatema, K., AshrafulHaq, J., Omar Faruq, M., Areef Ahsan, A.S.M., Golam Morshed, M.A.H., et al. 2010. Bacterial profile and their antimicrobial resistance pattern in an intensive care unit of a tertiary care hospital in Dhaka. Ibrahim Med. Coll. J., 4(2): 66-69.

Günserena, F., Mamıkog lua, L., Ztürkb, S., Yücesoyc, M., Biberog luc, K., Yulug c, N., et al. 1999. A surveillance study of antimicrobial resistance of Gram-negative bacteria isolated from intensive care units in eight hospitals in Turkey. J. Antimicrob. Chemother., 43: 373-378.

Zhang, Y. Mechanisms of Antibiotic Resistance in the Microbial World. Baltimore. Available from:www.moleculartb.org/gb/pdf/transcrip tions/11_YZhang.pdf.

Barai, L., Fatema, K., AshrafulHaq, J., Omar Faruq, M., Areef Ahsan, A.S.M., Golam Morshed, M.A.H., et al. 2010. Bacterial profile and their antimicrobial resistance pattern in an intensive care unit of a tertiary care hospital in Dhaka. Ibrahim Med. Coll. J., 4(2): 66-69.

\section{How to cite this article:}

Pampita Chakraborty and Sukumar Mukherjee. 2016. A Study on the Prevalence and Microbiological Profile of Nosocomial Infections in the ICU of a Tertiary Care Hospital in Eastern India. Int.J.Curr.Microbiol.App.Sci. 5(5): 920-925. doi: http://dx.doi.org/10.20546/ijcmas.2016.505.096 